\title{
Field-dependent collision frequency of the two-dimensional driven random Lorentz gas
}

\author{
Christoph Dellago* \\ Department of Chemistry, University of Rochester, Rochester, New York 14627 \\ Henk v. Beijeren \\ Institute for Theoretical Physics, University of Utrecht, Postbus 80006, Utrecht 3508 TA, The Netherlands \\ Debabrata Panja \\ Instituut Lorentz, Universiteit Leiden, Postbus 9506, 2300 RA Leiden, The Netherlands
}

J. R. Dorfman

Institute for Physical Sciences and Technology and Department of Physics, University of Maryland, College Park, Maryland 20742

(Received 8 May 2001; published 28 August 2001)

\begin{abstract}
In the field-driven, thermostated Lorentz gas the collision frequency increases with the magnitude of the applied field due to long-time correlations. We study this effect with computer simulations and confirm the presence of nonanalytic terms in the field dependence of the collision rate as predicted by kinetic theory.
\end{abstract}

DOI: 10.1103/PhysRevE.64.036217

In a recent paper [1], Panja, Dorfman, and van Beijeren used kinetic theory to derive analytic expressions for the Lyapunov exponents of the random, two-dimensional fielddriven Lorentz gas at moderately high densities. Their approach is based on the Bogoliubov-Born-Green-KirkwoodYvon hierarchy equations and takes into account correlated collision sequences. Such "ring collisions" lead to long-time tails in the Green-Kubo time correlations functions affecting the transport properties of the system [2]. Panja, Dorfman, and van Beijeren studied the effect of ring collisions on the Lyapunov exponents and found that long-time correlations cause a logarithmic dependence of the Lyapunov exponents on the applied field strength. These nonanalytic terms can be traced back to logarithmic terms in the field dependence of the collision frequency. Unfortunately, the nonanalytic contributions to the Lyapunov exponents are too small to be detected with current computer simulation techniques [3]. In contrast, the predicted effect on the collision frequency is large enough to be observed numerically. The purpose of this note is to verify the presence of logarithmic terms in the collision frequency with computer simulations.

The random, two-dimensional field-driven Lorentz gas, shown in Fig. 1, consists of a point particle of mass $m$ and charge $q$ moving under the influence of an external homogeneous field $\mathbf{E}$ in a two-dimensional array of circular nonoverlapping scatterers with radius $a$. The scatterers are fixed at random positions in the plane and have a density of $n$ $=N / A$, where $N$ is the total number of scatterers in the area $A$. In our numerical simulations we use periodic boundary conditions as indicated in Fig. 1. When the point particle leaves the simulation box through one specific boundary, it reenters it through the opposite boundary. Between collisions with the scatterers the point particle moves smoothly according to

\footnotetext{
*Corresponding author.

Email address: dellago@chem.rochester.edu
}

PACS number(s): 05.45.-a, 05.20.Dd, 05.20.-y

$$
\dot{\mathbf{r}}=\frac{\mathbf{p}}{m}, \quad \dot{\mathbf{p}}=q \mathbf{E}-\alpha \mathbf{p}, \quad \alpha=q \frac{\mathbf{E} \cdot \mathbf{p}}{p^{2}},
$$

where $\mathbf{r} \equiv\left\{r_{x}, r_{y}\right\}$ and $\mathbf{p} \equiv\left\{p_{x}, p_{y}\right\}$ are the position and the momentum of the moving particle, respectively. The second term in the momentum space part of the equations of motion is a Gaussian thermostat designed to remove the dissipated energy and keep the speed $v=p / m$ of the moving particle constant [4]. When the point particle collides with a scatterer it is reflected elastically, i.e.,

$$
\mathbf{v}_{+}=\mathbf{v}_{-}-2\left(\mathbf{v}_{-} \cdot \mathbf{u}\right)
$$

where $\mathbf{u}$ is the unit vector in the direction from the center of

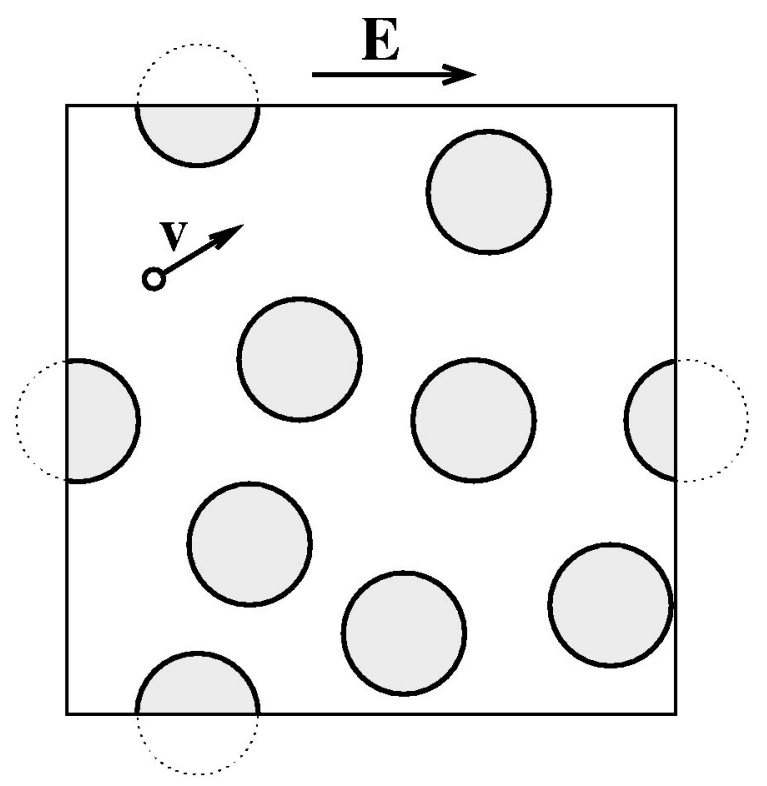

FIG. 1. Geometry of the random, field-driven Lorentz gas. 
the scatterer to the collision point and $\mathbf{v}_{-}$and $\mathbf{v}_{+}$are the preand postcollisional velocities of the moving particles, respectively.

In equilibrium, i.e., for $E=0$, and for long times, the phase distribution is uniform on the energy shell. In this case the collision frequency of the point particle with the scatterers can be obtained by determining the fraction of phase space available for collision in an infinitesimal time interval [5]

$$
\nu=\frac{2 n a v}{1-\pi n a^{2}} .
$$

In the presence of an external perturbation, however, the phase space distribution of nonequilibrium steady state collapses onto a multifractal strange attractor with information dimension strictly less than the phase space dimension. As a consequence, no analytical expression for the phase space distribution is available and the calculation of phase space integrals becomes cumbersome. Nevertheless, Panja, Dorfman and van Beijeren succeeded in determining the effect of the external field on the collision frequency by adopting a kinetic theory approach [1].

Qualitatively, it is evident that the external field increases the collision frequency. In the presence of the field, the velocity of the particle tends to be aligned in field direction before a collision. Immediately after the collision the velocity of the particle will therefore point against the field. Due to the action of the field the particle will turn around and possibly hit the same scatterer again after a time shorter than the average equilibrium collision time. The typical time scale for such a reorientation is of the order of $m v / q E$ [6]. Such correlated collisions enhance the collision frequency.

This argument was made quantitative by Panja, Dorfman, and van Beijeren [1]. As a consequence of correlated collisions, i.e., multiple collisions of the particle with the same scatterer, separated by sequences of intermediate collisions, a field dependent contribution appears in the collision frequency

$$
\nu=\frac{2 n a v}{1-\pi n a^{2}}+\frac{a \varepsilon^{2}}{2 \pi v} \ln \frac{2 n a v}{\varepsilon},
$$

where $\varepsilon=q|\mathbf{E}| /(m v)$. The first term on the right hand side of the above equation is the collision frequency in equilibrium. The second, nonanalytic field-dependent term is responsible for the nonanalytic dependence of the Lyapunov exponents on the field strength.

To verify the logarithmic term in Eq. (4) we have performed extensive simulations of the driven random Lorentz gas at various densities and field strengths. The collision frequency is obtained in a straightforward way by following the time evolution of the system for a long time and counting the number of collisions. For this purpose we use an analytical solution of equations of motion (1) and determine the collision point and the collision time of the moving particle with the scatterers numerically [4]. Typically, we study systems with $N=10^{5}$ scatterers in a square simulation box with peri-

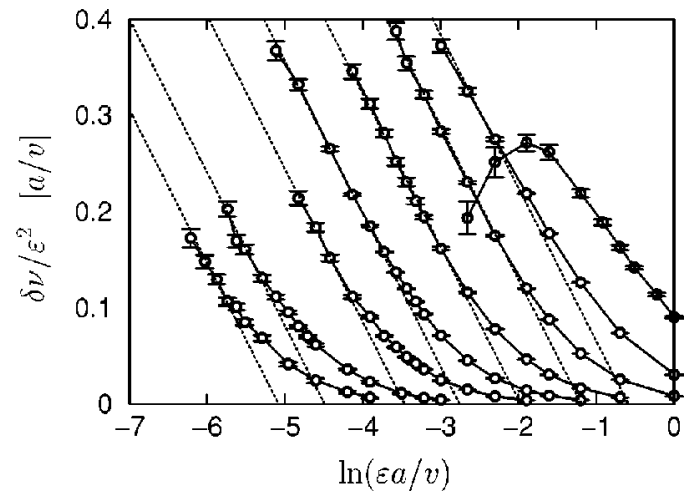

FIG. 2. The term $\delta \nu / \varepsilon^{2}$ as a function of $\ln (\varepsilon a / v)$ for the densities $n=0.001 a^{-2}, \quad 0.002 a^{-2}, \quad 0.005 a^{-2}, 0.01 a^{-2}, 0.02 a^{-2}$, $0.04 a^{-2}, 0.08 a^{-2}$, and $0.15 a^{-2}$ (from left to right). The dotted lines are straight lines with slope $-a /(2 \pi v)$ fitted to the data. The linear behavior of $\delta \nu / \varepsilon^{2}$ in the weak field regime indicates the presence of the logarithmic terms predicted by kinetic theory.

odic boundary conditions. Only at the highest density ( $n$ $=0.15 a^{-2}$ ) we use $N=5000$ scatterers. By averaging our results over various scatterer configurations we integrate over the quenched disorder of the randomly placed scatterers. Computing time is saved by dividing the simulation box into cells such that at each time only a few scatterers need to be considered as possible collision partners. For each density and field strength we typically carry out a total of more than $10^{9}$ collisions obtaining collision frequencies accurate to better than $0.01 \%$. This high accuracy is needed to detect the small changes in collision frequency in the weak field regime.

For analysis it is convenient to rewrite Eq. (4) as

$$
\frac{\delta \nu}{\varepsilon^{2}}=\frac{a}{2 \pi v} \ln \left(2 n a^{2}\right)-\frac{a}{2 \pi v} \ln \frac{\varepsilon a}{v},
$$

where $\delta \nu$ is the deviation of the collision frequency from its equilibrium value. Accordingly, $\delta \nu / \varepsilon^{2}$ should behave linearly when plotted as a function of $\ln (\varepsilon a / v)$. Figure 2 shows $\delta \nu / \varepsilon^{2}$ as a function of $\ln (\varepsilon a / v)$ for the densities $n$ $=0.001 a^{-2}, \quad 0.002 a^{-2}, \quad 0.005 a^{-2}, \quad 0.01 a^{-2}, \quad 0.02 a^{-2}$, $0.04 a^{-2}, 0.08 a^{-2}$, and $0.15 a^{-2}$. In Fig. 2 densities increase from left to right. Each data point is obtained as an average over 50-500 runs with different scatterer configurations. The error bars are estimated from the variation of the collision frequency in these sets of runs. The solid lines connect data points corresponding to the same density and the dotted lines are straight lines with slope $-a /(2 \pi v)$ fitted to the data in the low field regime.

For all densities but the highest one $\delta \nu / \varepsilon^{2}$ is a linear function of $\ln \varepsilon$ in the low field strength range with slope $-a /(2 \pi v)$ in agreement with Eq. (5). This confirms the existence of the nonanalytic field-dependent term predicted by kinetic theory.

From Fig. 2 we can also infer the range of validity of the theory in the $n-\varepsilon$ plane. Clearly, Eq. (5) breaks down for densities larger than about $n=0.08 a^{-2}$. For densities below $n=0.08 a^{-2}$ Eq. (5) holds for field strengths below a certain 
critical value $\varepsilon_{c}$. The intercept of the fitted lines with the $x$ axis can be used as a measure of this critical value. The critical value $\varepsilon_{c}$ is an approximately linear function of the density growing from $\varepsilon_{c} \sim 0.006 \mathrm{v} / a$ at $n=0.001 a^{-2}$ to $\varepsilon_{c}$ $\sim 0.5 \mathrm{v} / a$ at $n=0.08 a^{-2}$.

However, the agreement between numerical results and analytical theory is not perfect even for low densities and weak external fields. While the lines corresponding to Eq. (5) have the correct slope, their intercept with the $y$ axis is lower by about $0.2 a / v$ than the value obtained numerically. This discrepancy is essentially constant in the density range we have studied. It must be due to the approximation of the probability of return to a given scatterer by a solution of the diffusion equation, as described in the Appendix of Ref. [1]. In order to check this in detail one would have to explicitly solve the Lorentz-Boltzmann equation in presence of the field and thermostat.

In summary, we have unequivocally detected the appearance of nonanalytic terms in the collision frequency and hence, indirectly, in the Lyapunov exponents of the random field-driven Lorentz gas. Our numerical results agree quantitatively with the kinetic theory predictions of Panja, Dorfman, and van Beijeren.

J.R.D. thanks the National Science Foundation for support under Grant No. PHY-98-20824.
[1] D. Panja, J. R. Dorfman, and H. van Beijeren, J. Stat. Phys. 100, 279 (2000).

[2] J. R. Dorfman and H. van Beijeren, Statistical Mechanics, Part B. Time-dependent Processes, edited by Bruce J. Berne (Plenum, New York, 1977).
[3] C. Dellago and H. A. Posch, Phys. Rev. Lett. 78, 211 (1997).

[4] B. Moran and W. G. Hoover, J. Stat. Phys. 48, 709 (1987).

[5] J. Machta and R. Zwanzig, Phys. Rev. Lett. 50, 1959 (1983).

[6] C. Dellago, L. Glatz, and H. A. Posch, Phys. Rev. E 52, 4817 (1995). 